\title{
Characterization of Element Partitioning at the Austenite/Ferrite Interface of as Cast CF-3 and CF-8 Duplex Stainless Steels
}

\author{
R. Prakash Kolli ${ }^{1}$, Sarah Mburu ${ }^{1}$, Daniel E. Perea ${ }^{2}$, Jia Liu $^{2}$, Samuel C. Schwarm ${ }^{1}$, Arielle Eaton ${ }^{2}$, \\ Sreeramamurthy Ankem ${ }^{1}$ \\ 1. Department of Materials Science and Engineering, University of Maryland, College Park, USA. \\ 2. Environmental and Molecular Sciences Laboratory, Pacific Northwest National Laboratory, Richland, \\ USA.
}

Cast duplex stainless steels are used in cooling water piping of light water reactors (LWRs) due to their combination of strength, high ductility, high impact toughness, corrosion resistance, castability and weldability. This combination is due to the presence of the face-centered cubic (f.c.c.) $\gamma$-austenite and body-centered cubic (b.c.c.) $\alpha$-ferrite phases in the duplex microstructure. The ferrite phase is present in volume fractions less than approximately $20 \%$ in grades used in LWR power plants. These alloys experience thermal aging embrittlement during operational service due to spinodal decomposition of the $\alpha$-ferrite phase into chromium-rich (Cr-rich) $\alpha$-martensite and iron-rich (Fe-rich) $\alpha$-ferrite phases, and possibly nucleation and growth of intermetallic G-phase precipitates or carbides [1,2]. Microstructural evolution and concomitant changes in macroscopic (bulk) mechanical properties lead to increased hardness, a loss of ductility, and a reduction in impact toughness.

Interest in extending the operational life of these power plants to 80 years requires examining the complex phase decomposition and corresponding mechanical property changes of these stainless steels by employing accelerated isothermal aging at elevated temperatures relative to the operational temperatures. This requires detailed compositional characterization of the as cast stainless steels in order to provide a baseline reference to quantify the temporally evolving concentration profiles and phase decomposition at the different temperatures. Additionally, the $\gamma$-austenite $/ \alpha$-ferrite heterophase interface must be characterized, as possible heterogeneous nucleation and growth of intermetallic precipitates or carbides at this location will significantly influence the local elemental concentration profiles and bulk mechanical properties. Thus, we have characterized the statically cast $\mathrm{CF}-3,[\mathrm{Fe}, 0.02$ C, 1.07 Mn, 0.98 Si, 19.69 Cr, 8.4 Ni, 0.28 Mo (wt.\%)], and CF-8, [Fe, 0.06 C, 0.99 Mn, 0.97 Si, 19.85 $\mathrm{Cr}, 8.3 \mathrm{Ni}, 0.35 \mathrm{Mo}$ (wt.\%)] duplex stainless steel microstructures at two different length scales employing state-of-the-art atom-probe tomography (APT) and energy-dispersive x-ray spectroscopy (EDS). We perform initial characterization of the concentration profiles with the proximity histogram [3] relative to a $\mathrm{Ni}$ isoconcentration surface, Figure 1, to illustrate element enhancement in the constituent phases. We illustrate a procedure to quantify the phase compositions from the proximity histogram. The preliminary elemental partitioning ratio of each element $i, \kappa_{i}^{\alpha / \gamma}=C_{i}^{\alpha} / C_{i}^{\gamma}$, where $C_{i}^{j}$ is the concentration of element $i$ in phase $j$, at the $\gamma$-austenite $\alpha$-ferrite heterophase interface is calculated. Additionally, we quantify the phase compositions employing elemental maps of each phase from the EDS spectra, Figure 2, and also calculate the quantity $\kappa_{i}^{\alpha / \gamma}$. Our initial results indicate that the $\alpha$-ferrite phase is enriched in $\mathrm{Cr}$, whereas the $\gamma$-austenite phase is enriched in Fe and nickel (Ni). Additionally, the high spectral and spatial resolution of the APT technique illustrates that the $\alpha$-ferrite phase is enriched in silicon (Si) and molybdenum (Mo), whereas the $\gamma$-austenite phase is enriched in manganese (Mn). We also illustrate that the carbon (C), boron (B), and phosphorus (P) are enhanced at the $\gamma-$ austenite $/ \alpha$-ferrite heterophase interface. In addition, we discuss local enhancement and depletion of $\mathrm{Fe}$ and $\mathrm{Cr}$ at the interface, which is possibly due to diffusion gradients or to local magnification effects. 


\section{References:}

[1] HM Chung and TR Leax, Mater. Sci. Tech., 6 (1990), p. 249.

[2] F Danoix and P Auger, Mater. Charact., 44 (2000), p. 177.

[3] OC Hellman, JA Vandenbroucke, J Rüsing, D Isheim and DN Seidman, Microsc. Microanal., 6 (2000), p. 437.

[4] The authors acknowledge funding from the Department of Energy, Nuclear Energy University Program, Dr. Jeremy Busby, technical monitor.
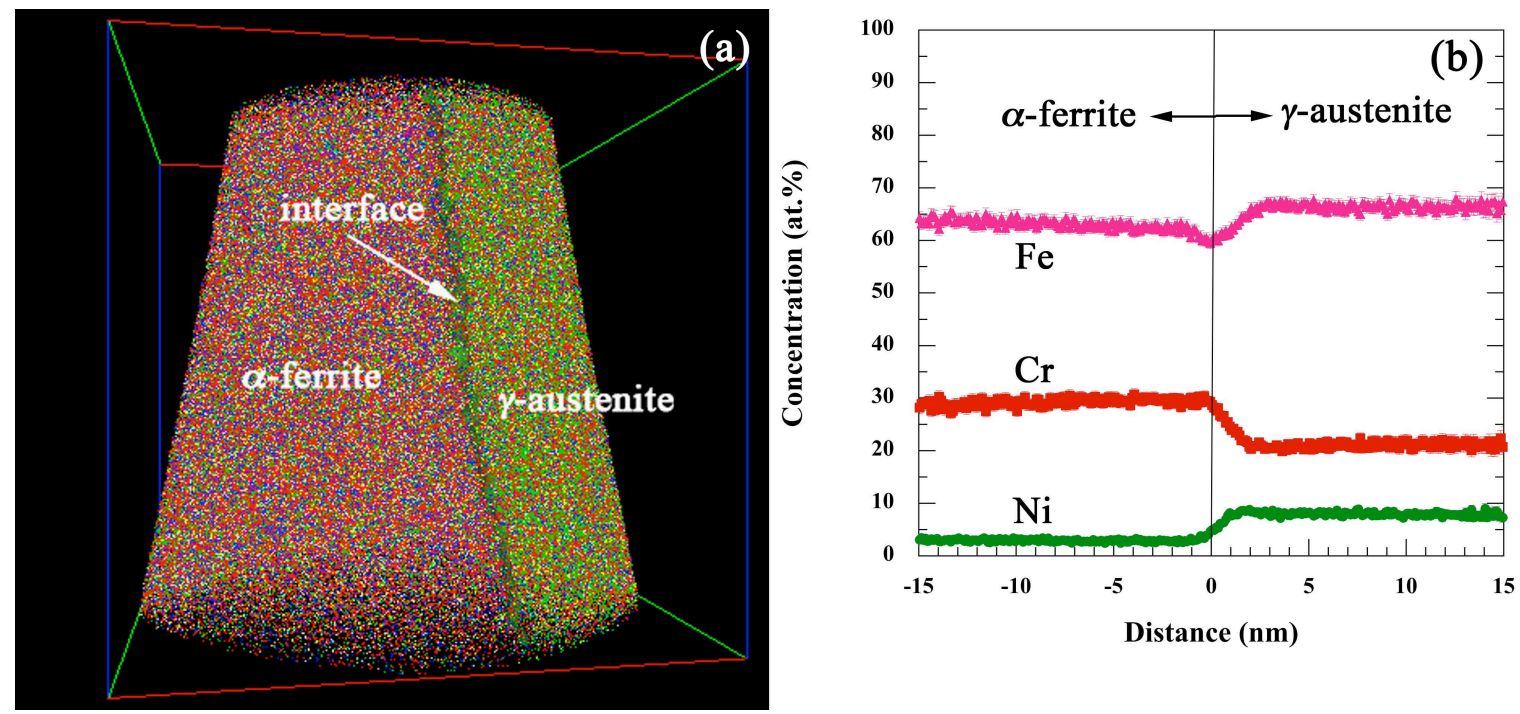

Figure 1. The $\gamma$-austenite $/ \alpha$-ferrite heterophase interface delineated by a 4.5 at. $\%$ Ni isoconcentration surface in (a) an atom probe tomography (APT) reconstruction, and (b) the corresponding proximity histogram concentration profiles for $\mathrm{Fe}$ (pink), $\mathrm{Cr}$ (red), and $\mathrm{Ni}$ (green) in a $\mathrm{CF}-8$ as cast duplex stainless steel. The ca. $60 \times 60 \times 60 \mathrm{~nm}^{3}$ reconstruction contains $\sim 5.0$ million ions. The $\pm 2 \sigma$ error bars are based on counting statistics.

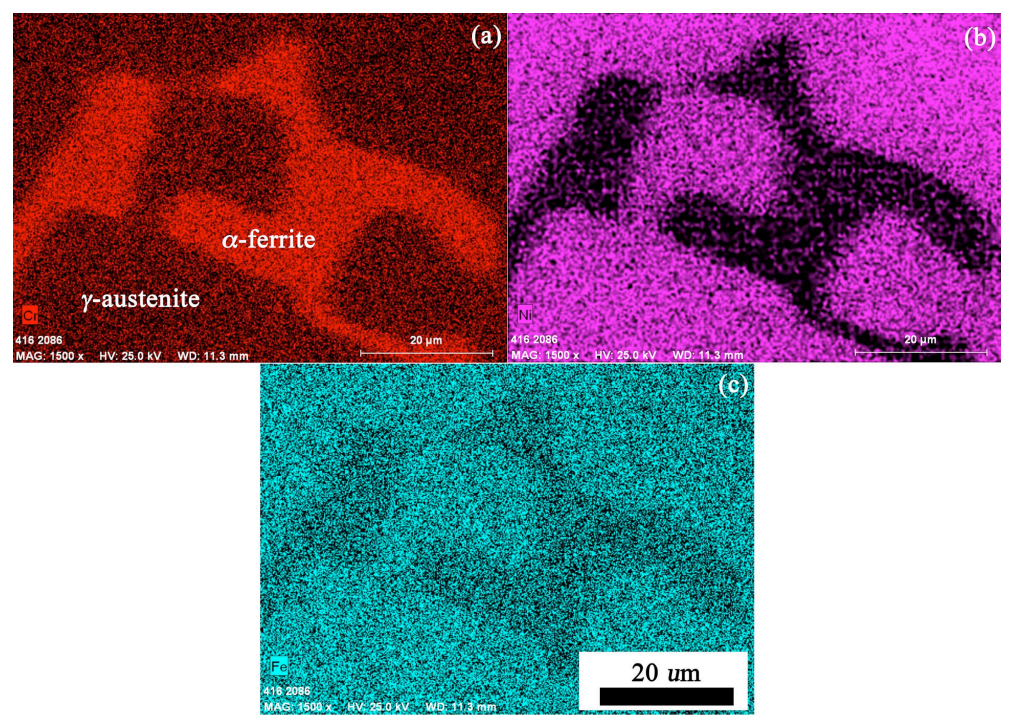

Figure 2. Energy-dispersive X-ray spectroscopy (EDS) elemental maps for (a) Cr (red), (b) Ni (pink), and (c) Fe (teal) of the CF-3 as cast duplex stainless steel illustrating $\mathrm{Cr}$ enrichment in the $\alpha$-ferrite phase, and Fe and Ni enrichment in the $\gamma$-austenite phase. 